\title{
Stage III Choroidal and Ciliary Body Melanoma AJCC v8
}

National Cancer Institute

\section{Source}

National Cancer Institute. Stage III Choroidal and Ciliary Body Melanoma A/CC v8. NCI Thesaurus. Code C140664.

Stage III includes: IIIA (T2c-d, N0, M0); (T3b-c, N0, M0); (T4a, NO, M0); IIIB (T3d, N0, M0); (T4b-c, N0, M0); IIIC: (T4d-e, N0, M0). T2c: T umor size category 2 without ciliary body involvement but with extraocular extension less than or equal to $5 \mathrm{~mm}$ in largest diameter. T2d: Tumor size category 2 with ciliary body involvement and extraocular extension less than or equal to $5 \mathrm{~mm}$ in largest diameter. T3b: Tumor size category 3 with ciliary body involvement. T3c: T umor size category 3 without ciliary body involvement but with extraocular extension less than or equal to $5 \mathrm{~mm}$ in largest diameter. T4a: Tumor size category 4 without ciliary body involvement and extraocular extension. T3d: Tumor size category 3 with ciliary body involvement and extraocular extension less than or equal to $5 \mathrm{~mm}$ in largest diameter. T4b: T umor size category 4 with ciliary body involvement. T4c: T umor size category 4 without ciliary body involvement but with extraocular extension less than or equal to $5 \mathrm{~mm}$ in largest diameter. T4d: Tumor size category 4 with ciliary body involvement and extraocular extension less than or equal to $5 \mathrm{~mm}$ in largest diameter. T4e: Any tumor size category with extraocular extension more than $5 \mathrm{~mm}$ in largest diameter. N0: No regional lymph node metastasis. M0: No distant metastasis. (AJCC 8th ed.) 